\title{
APPLICATIONS OF RESONANCE IONIZATION SPECTROSCOPY TO ULTRALOW-LEVEL COUNTING AND MASS SPECTROSCOPY*
}

\author{
S D KRAMER, G S HURST, J P YOUNG, M G PAYNE, M K KOPP, \\ T A CALLCOTT**, E T ARAKAWA, and D W BEEKMAN**
}

\author{
Oak Ridge National Laboratory, Oak Ridge, Tennessee 37830
}

ABSTRACT. In this paper it is shown that the ability to directly detect a daughter atom, using resonance ionization spectroscopy, in delayed time coincidence with the decay of a parent species promises to drastically reduce the background in low-level counting experiments. In addition, resonance ionization can also be used as an ion source for a mass spectrometer system that is capable of discriminating between isobars.

Significant improvement in the sensitivity of low-level counting can be achieved by application of the technique of Resonance Ionization Spectroscopy (RIS). The RIS technique is a photo-ionization method in which atoms of a given quantum selected species, in a gas phase, are converted to ion pairs by absorption of photons from precisely tuned lasers (Hurst and others, 1979a). The technique is capable of elemental specificity and can detect single atoms since devices such as proportional counters and electron multipliers can easily detect single charged particles. In this paper, two possible practical configurations of RIS are discussed which, when perfected, should have important applications in several areas.

The first example describes a method that can significantly reduce the background in a low-level counting experiment. This is done by requiring both a radioactive counting event and the RIS detection of the expected recoil daughter atom to be in time and space coincidence to insure a valid detection signal. In the second application, RIS is being studied as an ionization source for a mass spectrometer. Since RIS selectivity is principally based on atomic number while the selectivity of a mass spectrometer is due to atomic mass, the combination of both of these techniques should significantly reduce the background due to isobars and other causes in many situations.

The basic principle of RIS is shown schematically in figure 1. An atom in its ground state, 1, can be easily excited to a higher energy state, 2 , if it is immersed in a flux of photons whose energy, $h \omega$, equals the effective energy difference between the two states, $\Delta \mathrm{E}_{12}$. However, if $h \omega$ $\neq \Delta \mathrm{E}_{12}$, the atom will remain essentially in its ground state. If the photon flux is made high enough, the induced transition rate will be much higher than the spontaneous decay, $\Gamma_{21}$. In this case, the probability of finding an atom in its excited state, 2 , is determined by the statistical weights of states 1 and 2. In this example, another photon of the same energy can then efficiently ionize an atom in state 2 but not in state 1 . If two conditions are met, all of the atoms can be ionized by the process just described. The first condition is that the product of the photon energy flux, $\mathrm{F}$, times the state 2 photo-ionization cross section, $\sigma_{I}$, is much larger than the rate, $\beta$, at which the intermediate state is destroyed by possible

* Research sponsored by the US Department of Energy under contract W-7405eng-26 with the Union Carbide Corporation

** University of Tennessee, Knoxville, Tennessee 37916 
competing processes that convert it to a state that cannot be ionized. This insures that ionization is the dominant process. The second condition is that $\sigma_{\mathrm{I}} \mathrm{F} \tau>>1$, where $\tau$ is the time the photon pulse is on. For typical values of $\sigma_{\mathrm{I}}$, this requirement is met when $\mathrm{F} \tau \sim 0.1$ joule $/ \mathrm{cm}^{2}$ which is a value that can easily be achieved with lasers over areas of about $1 \mathrm{~cm}^{2}$. When both conditions are met, in which case the neutral ground state atom is converted with essentially 100 percent efficiency to an electron-ion pair, the RIS process is said to be saturated. Since the probability of ionization is very small if $h \omega \neq \Delta \mathrm{E}_{12}$, the process is selective since the laser can be tuned to excite an energy level present only in the species of interest. Using slightly more complicated schemes that depend on more than one intermediate state, saturated RIS can be used to ionize and, thus, detect all the atoms in the periodic table except F, He, Ne, and $\mathrm{Ar}$ which have first excited states too high in energy to be reached by commercially available lasers.

In order to demonstrate the usefulness of RIS in low-level counting, it is necessary to show that it is possible to detect a single specific daughter atom in close time coincidence with the decay of the parent. The first experiment to demonstrate this is shown in figure 2 (Kramer and others, 1978). A weak (1 fission/second) ${ }^{252} \mathrm{Cf}$ source was used. The ${ }^{252} \mathrm{Cf}$ undergoes asymmetric fission, and it is known that 16 percent of the atoms in the heavy fission fragment will consist of Cs. The particle detector senses the presence of a fission fragment by energy discrimination and provides a signal that the complementary fragment is heading away from the detector. As this highly charged and very energetic fragment $(>40 \mathrm{MeV})$ is slowed to thermal energies by collision with other atoms or molecules in the buffer gas composed of 90 percent $\mathrm{Ar}$ and 10 percent $\mathrm{CH}_{4}$, it causes the ejection of approximately $2 \times 10^{6}$ electrons. However, the track of the fission fragment is arranged to be between two charged plates polarized so that the electrons are drifted to the positive plate away from the proportional counter. After collection, the potential on the plates is reversed so that any additional electrons produced by the 400-nsec laser pulse will drift into the proportional counter. With the apparatus configuration used, we expected to detect 7 Cs atoms per 100 fissions if detection proved to be 100 percent efficient. With the laser tuned to excite the

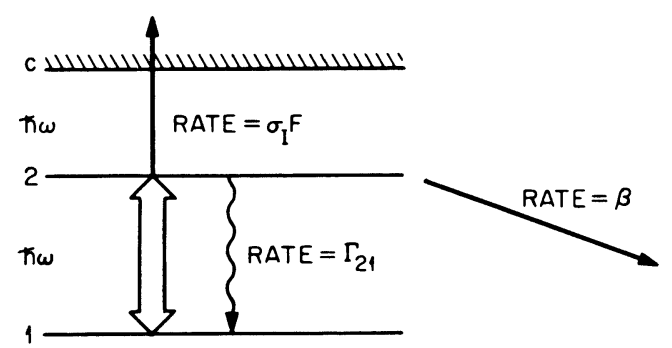

Fig 1. Schematic diagram for the RIS process in which the final ionization state (1) can be ionized by first exciting it to another bound state (2), making ionization a resonance process. The symbols are explained in the text. 
$7 \mathrm{P}_{3 / 2}$ or $7 \mathrm{P}_{1 / 2}$ Cs intermediate states and after eliminating a background signal due to probable negative ion formation, $8 \pm 2$ detection events $/ 100$ fissions were observed, as shown in figure 3 . These results imply that the highly charged Cs fission fragment neutralizes in the buffer gas. This process of neutralization depends in detail on the composition of the buffer gas and the energy of the ion.

A common technique used in low-level counting is based on detecting the decay of a radioactive parent nucleus in a proportional counter (Oeschger and Wahlen, 1975). This has proved quite useful since, with some care, the detection efficiency for an event can often even approach 100 percent. The problem, therefore, is not so much in actually detecting the decay event of interest as it is in separating a real event from the virtually inevitable background events that are present. Reduction in background has been achieved by using shielding materials, anti-coincidence techniques, pulse risetime analysis, and multiple decay schemes. The last two methods, however, are useful only in special cases. In contrast, RIS is applicable to virtually all of the elements; and, so, its use in selectively detecting the daughter product of a radioactive process is quite general. The main advantage in using this technique is that it turns an ordinary counting experiment into a two-event coincidence experiment with its concomitant reduction in background.

Figure 4 shows schematically the fundamental principles involved in using daughter atom detection to reduce background. A proportional counter designed with a wire offset to one side is used. This is done so that a large fraction of the counter volume can be swept out by a pulsed laser beam. At Oak Ridge, a counter of this type has been found to work quite satisfactorily. The counter is filled with a suitable counting gas and also with a small amount of a gaseous species that contains the radioactive parent of interest. The absolute amount of the radioactive species should

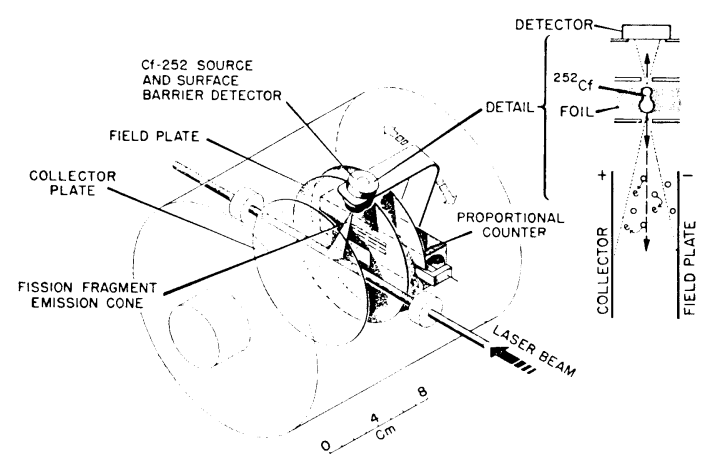

Fig 2. Schematic drawing of the apparatus used to detect a single atom created by spontaneous fission. A signal generated in the surface-barrier detector indicates the occurrence of a single fission of ${ }^{252} \mathrm{Cf}$. After the initial track ionization has been swept onto the collector plate, a tunable laser pulse is used to photoionize the selected single atom. The liberated photoelectron then drifts into the proportional counter and is detected as a single event. 
be small enough so that under the operating conditions of the counter it is entirely in the gas phase.

It should be stressed that the gaseous radioactive species can be molecular since it is known that in many types of decay processes of interest, particularly those that result in Auger cascades, the daughter atom is freed from its molecular host. However, it may be freed as an ion and if so, it must be neutralized in order to carry out RIS analysis of the daughter. This requirement rests on the fact that the energy levels of virtually all ions are too high in energy to saturate with currently available lasers. There exist many possible ways to neutralize ions. Perhaps the most straightforward is to mix the counting gas with a species that has an ionization potential smaller than that of the daughter atom so that electron transfer can take place from the "donor" species to the positive ions of interest. With suitable donor densities, the neutralization time can possibly be made shorter than the laser pulse length. This has the ad-

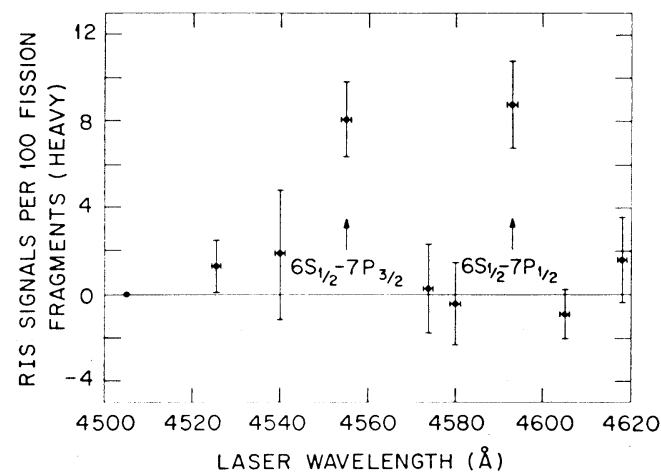

Fig 3. Experimental results showing that it is possible to detect single daughter atoms. The data points at the indicated wave lengths represent the number of photoelectron events per 100 heavy-mass fission fragments counted in the surface barrier detector. These points are taken from the raw experimental results after a small background effect due to probable negative ion formation in the track was eliminated.

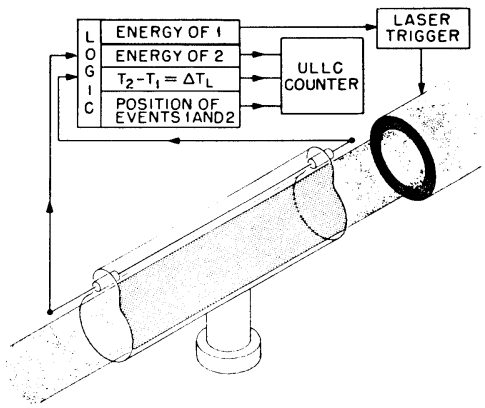

Fig 4. Proportional counter designed for RIS detection of single daughter atoms. The displaced wire permits sweeping of a large fraction of the counter volume with the laser beam. A valid ultralow-level count (ULLC) occurs only if the energies, times, and positions of the initial parent decay signal and the subsequent daughter RIS signal meet the required conditions explained in the text. 
vantage that the daughter ion could then be ionized and neutralized many times during a single laser pulse; and, so, the RIS signature would be due to many electrons rather than a single electron. Other schemes for achieving RIS with amplification, RISA, have also been proposed (Hurst and others, 1979b).

When a rare decay or background event occurs, the signal from the counter will be used to trigger-after a short time delay to allow the counter to recover-a laser system tuned to the appropriate wave lengths necessary to ionize the expected daughter atom. If a real decay of interest occurs, the signal due to the ionization of the daughter atom by the tuned laser will be detected in delayed-time coincidence with the decay of the parent. Virtually all other types of decay or sources of background will not produce the designated daughter atom and, thus, will not yield a second coincidence signal when the laser is fired. By including in the buffer gas or on the walls of the counter a substance that will react with the daughter, the counter can be cleared between decays to avoid multiple counting of the same daughter atom. Alternatively, the counter can be designed such that the daughter remains in the vapor phase and the change in signal amplitude with the number of decays can be monitored.

By using a high resistance counter wire the longitudinal position of the event, in addition to its energy, can be measured. Therefore, by using a position-sensitive proportional counter (as shown in fig 4), the following conditions must be met simultaneously for a valid decay to be measured: 1) the measured energy of the initial decay event must be appropriate for that of the desired parent atom, 2) there must be a signal of appropriate amplitude at the time the laser is fired, 3) both events must occur at the same position in the counter.

By requiring all these conditions, background events can be virtually eliminated. Consider the case of a well-shielded counter with a background count rate of 1 count/minute. Using a laser with a pulse length of $1 \mu \mathrm{s}$ (which also corresponds to about the counter recovery time) and using only the time coincidence requirement, the effective background will be reduced to about $10^{-8}$ counts/minute. One possible effect that must be avoided is the production of spurious electrons in time coincidence with the laser pulse due to causes independent of the presence of the daughter atom. This possible source of background could be due to off-resonance multiphoton ionization of gaseous species or to surface photoelectron ejection due to scattered laser light hitting the chamber

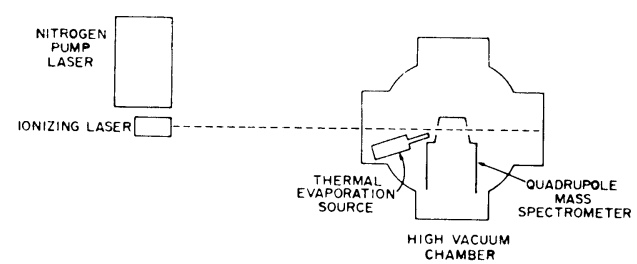

Fig 5. Schematic drawing of the apparatus used to demonstrate the use of RIS as a source for a mass spectrometer. 
walls. However, single atoms of Cs have been detected in gas mixtures containing up to $10^{19}$ other atoms or molecules $/ \mathrm{cm}^{3}$. Thus, in many cases, this will not be a hindrance (Hurst and others, 1979a). A rather conservative estimate would be that there is only a 1 percent chance for the laser pulse to produce an electron in the absence of the daughter atom. In addition, making the conservative estimate that the position resolution of the counter is 1 part in 100 , there would be only a 1 percent chance that the parent decay and the spurious electron would occur at the same longitudinal position in the counter. Taken together, the effective background count rate using RIS would, thus, be a factor of $10^{-4}$ below that of an identical counter not using the laser coincidence technique.

A possible application of this technique currently under active development is the detection of ${ }^{7} \mathrm{Be}$ produced by the interaction of solar neutrinos with ${ }^{7} \mathrm{Li}$ (Bahcall, 1978). In this project, approximately 100 atoms of ${ }^{7} \mathrm{Be}$ in the form of a volatile organic ${ }^{7} \mathrm{Be}$-containing compound will be placed in a proportional counter for a time comparable to its half-life of 53 days. Beryllium-7 decays by electron capture to ${ }^{7} \mathrm{Li}$, and 90 percent of the time the sole decay signature is a $50-\mathrm{eV}$ electron. This is a poor decay signature for traditional background discrimination, but it is adequate to trigger the laser to detect the expected daughter ${ }^{7} \mathrm{Li}$. Other possible applications of the technique are in detecting tracer ${ }^{39} \mathrm{Ar}$ in the environment (Oeschger and Wahlen, 1975) and in cosmochronology (Kirsten, 1978).

Another application of RIS has been in the development of a source for a mass spectrometer system capable of making isotopic ratio measurements of solid samples (Janes and others, 1976; Beekman and others, ms in preparation). If the RIS process is saturated for all isotopes of an element, then the ionized atoms will be an accurate isotopic representation of the sample for the element under investigation. Such a system would combine the excellent chemical (atomic number) selectivity of the RIS process with the mass selectivity of a mass spectrometer to enable one to make isotopic ratio measurements without the usual interference due to isobars. For example, in a ${ }^{14} \mathrm{C}$ measurement, the ionization probability

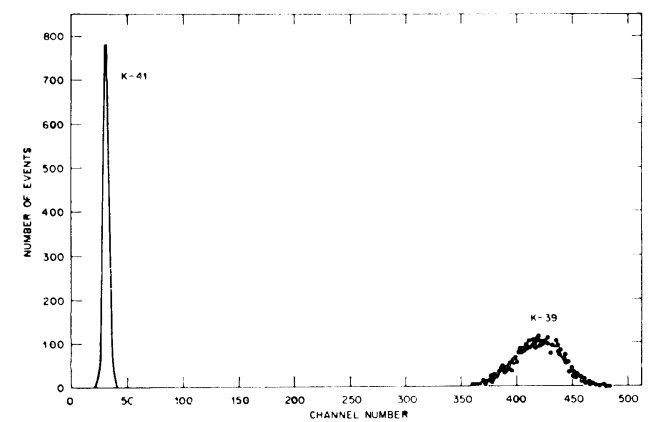

Fig 6. Pulse height spectrum of the output of a mass spectrometer which used a RIS source to generate ${ }^{39} \mathrm{~K}$ and ${ }^{41} \mathrm{~K}$ ions. The channel number is directly proportional to the number of ions detected. 
for ${ }^{14} \mathrm{C}$ would be 1 (by design), and the ionization probability for ${ }^{14} \mathrm{~N}$ could be as low as $10^{-14}$.

Figure 5 shows a system used to demonstrate the principle of resonance ionization as an ion source for mass spectroscopy (Beekman, ms in preparation). The thermal evaporation source provided a loosely collimated beam of neutral potassium atoms in a vacuum. In other experiments a focused laser was used to eject atoms from the surface. This method would be particularly useful when dealing with refractory materials or molecules. In addition, use of laser ablation should eliminate most of the normally required sample preparation. This is true since the chemical form of the solid sample is unimportant. Another valuable feature would be to use the laser to remove surface contamination before making actual measurements on the virgin underlying material. The ionization laser pulse, which in this case was not strong enough to saturate the transition, intersected the beam of potassium neutral just over the entrance aperture to a quadrupole mass analyzer. On successive firings of the ionizing laser, the spectrometer was alternately tuned to mass/charge ratios of 39 and 41 .

The analyzed signals from the mass analyzer are shown in figure 6 . The first peak represents the ${ }^{41} \mathrm{~K}$ pulse heights, and the second peak corresponds to the larger ${ }^{39} \mathrm{~K}$ signals. The spread in pulse heights $(\sim 20$ percent FWHM in each peak) is due to variations in the thermal vaporization source and in the ionizing laser output. An isotope ratio was calculated using the signal amplitude of the maxima. The value found for the ${ }^{39} \mathrm{~K}:{ }^{41} \mathrm{~K}$ ratio was $13.6: 1$, which is in good agreement with the accepted value of 13.5:1.

Currently under development is a time-of-flight mass spectrometer (although a Mattauch design is also under consideration and, of course, various accelerators could also be used) that will employ a laser capable of saturating the RIS process as an ion source. This will increase the sensitivity and resolution above that which could be obtained using the simple apparatus described.

In this paper the application of RIS to low-level counting and isotope differentiation has been described. Although rather new, it appears that this technique can make an important contribution to research in both of these areas.

RFFERFNCES

Bahcall, J N, 1978, Solar ncutrino experiments: Rev Modern Physics, v 50, p 81-903.

Beekman, D W, Callcott, T A, Kramer, S D, Arakawa, E T, Hurst, G S, and Nussbaum, E, in press, Resonance ionization source for mass spectroscopy: Internatl Jour Mass Spectrometry Ion Physics, in press.

Hurst, G S, Payne, M G, Kramer, S D, and Young, J P, 1979a, Resonance ionization spectroscopy and one-atom detection: Rev Modern Physics, v 51, p 767-819.

1979b, Resonance ionization spectroscopy with amplification: Chem Physics Letters, v $63, \mathrm{p} 1-4$.

Janes, G S, Itzkan, I, Pike, C T, Levy, R H, and Levin, L, 1976, Two photon laser isotope separation of atomic uranium: Jour Quantitative Electronics, v 12, p 111-120.

Kirsten, T, 1978, Time and the solar system, in Dermott, S F, ed, Origin of the solar system: London, John Wiley \& Sons, $79 \mathrm{p}$.

Kramer, S D, Bemis, Jr, C E, Young, J P, and Hurst, G S, 1978, One-atom detection in individual ionization tracks: Optics Letters, v 3, p 16-18.

Oeschger, Hans and Wahlen, M, 1975, Low level counting techniques: Ann Rev Nuclear Sci, v 25, p 423-463. 